\title{
A Rare Cause of Abdominal Compartment Syndrome: Chylous Ascites in non-Hodgkin's Lymphoma
}

ISSN: 2639-0531

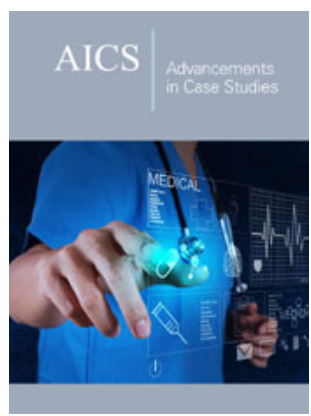

*Corresponding author: Marjan Alidoost, Department of Medicine, USA

Submission: 毕 November 25, 2019

Published: 眥December 05, 2019

Volume 2 - Issue 2

How to cite this article: Marjan Alidoost, Conte GA, Patel I, Farooq T, Dada 0, et al. A Rare Cause of Abdominal Compartment Syndrome: Chylous Ascites in nonHodgkin's Lymphoma. Adv Case Stud.2(2) AICS.000535.2019.

DOI: 10.31031/AICS.2019.02.000535

Copyright@ Marjan Alidoost, This article is distributed under the terms of the Creative Commons Attribution 4.0 International License, which permits unrestricted use and redistribution provided that the original author and source are credited.

\author{
Marjan Alidoost ${ }^{1 *}$, Conte GA ${ }^{1}$, Patel $\mathrm{I}^{1}$, Farooq $\mathrm{T}^{2}$, Dada $\mathbf{0}^{1}$, Miskoff $\mathrm{JA}^{1}$ and \\ Levitt $\mathbf{M J}^{1}$ \\ ${ }^{1}$ Department of Medicine, USA \\ ${ }^{2}$ Department of Pathology, USA
}

\begin{abstract}
Background: Abdominal compartment syndrome (ACS) occurs in critically sick patients and is defined as intra-abdominal pressure (IAP) over $20 \mathrm{mmHg}$ accompanied by new-onset organ dysfunction [1]. ACS necessitates emergent therapy to decrease abdominal pressures-whether it be via surgical means, or in the case of our patient, paracentesis [1].
\end{abstract}

Case presentation: A 60-year-old male with a medical history of coronary artery disease status post coronary artery bypass graft and hypertension presented to the Emergency Department (ED) with shortness of breath of 2 days duration preceded by three weeks of increasing abdominal pain and lower extremity edema. He was found to have large ascites and extensive lymphadenopathy on computed tomography (CT) of the abdomen and pelvis. He went for emergent paracentesis and $13,500 \mathrm{~mL}$ of chyle was removed from the peritoneum. He was eventually diagnosed with non-Hodgkin's lymphoma (NHL) and started on chemotherapy. Unfortunately, he expired four months later due to a cardiac arrest at home.

Conclusion: Although ACS is more classically caused by trauma and/or bleeding, severe liver cirrhosis, ileus, excessive fluid resuscitation or transfusion, it can also be caused by any process that increases IAP [1]. ACS has been reported due to a retroperitoneal mass from NHL but has not yet been described as a complication of malignant chylous ascites [2]. Here, we report the first case of ACS caused by chylous ascites in the setting of newly diagnosed NHL

Keywords: Abdominal compartment syndrome (ACS); Non-Hodgkin's lymphoma (NHL); Chylous ascites; Malignancy

\section{Introduction}

Abdominal compartment syndrome (ACS) carries a significant morbidity and mortality. It typically affects surgical patients, such as trauma patients, burn patients, and patients with recent abdominal surgery [1]. However, it carries a significantly worse mortality in nonsurgical patients and requires critical attention to these patients. ACS is diagnosed as intraabdominal pressure (IAP) over $20 \mathrm{mmHg}$ with accompanying new onset organ failure [1]. Even with treatment, mortality is high in patients with ACS [1]. Signs and symptoms include abdominal pain, shortness of breath, tachypnea, acute renal failure and bowel ischemia [1]. ACS can also be a life-threatening manifestation of cirrhosis, malignancy, acute pancreatitis or retroperitoneal bleed [2]. In our case, the patient had increasing abdominal girth over three weeks and presented to the Emergency Department with sudden onset of abdominal pain and dyspnea. He underwent emergent paracentesis with 13 liters of chylous fluid drained and was eventually diagnosed with stage IV follicular lymphoma. This case reports a rare presentation of ACS due to chylous ascites in the setting of follicular lymphoma. We highlight the importance of pursuing a through workup for causes of chylous ascites as well as understanding the underlying mechanisms and management of ACS.

\section{Case Report}

A 60-year-old male from New Jersey, United States with a medical history of coronary artery disease status post coronary artery bypass graft $\mathrm{x} 4$, hypertension, hyperlipidemia presented to the Emergency Department (ED) with shortness of breath. Three weeks 
prior to presentation he began to develop edema of his lower extremities and abdomen. He also reported associated malaise, easy fatigability, and dyspnea upon exertion. During the 48 hours preceding admission, his abdominal distension had worsened significantly, and he developed sudden shortness of breath at rest and thus presented to the ED. At baseline he could ambulate without difficulty and had never experienced similar symptoms. In the ED, Computed Tomography (CT) of the abdomen and pelvis was performed which revealed diffuse lymphadenopathy, large amount of ascites, along with splenomegaly (Figure 1). Of note, on his admission laboratory investigations, he was found to have a white blood cell count (WBC) of $69.9 \mathrm{~K} / \mathrm{uL}$, creatinine of $5.26 \mathrm{mg} / \mathrm{dL}$ and a potassium of $6.9 \mathrm{mmol} / \mathrm{L}$ (Table 1 ). The patient was afebrile and blood cultures drawn from two different peripheral sites were drawn and resulted negative. Thus, patient was not found to be in sepsis and infectious etiology was ruled out. He was determined to be in acute renal failure and respiratory distress secondary to abdominal compartment syndrome, suspected to be caused by malignant ascites. He then went to Interventional Radiology for emergent paracentesis where $13,500 \mathrm{~mL}$ of milky peritoneal fluid was removed and sent for analysis. A temporary dialysis catheter was also placed in his internal jugular vein for one session of emergent dialysis. Initially, tumor lysis syndrome was also on the differential, given his new-onset renal failure with hyperkalemia and elevated uric acid. However due to tense abdomen, end-organ damage and significant drainage from his paracentesis, he was clinically diagnosed with abdominal compartment syndrome.

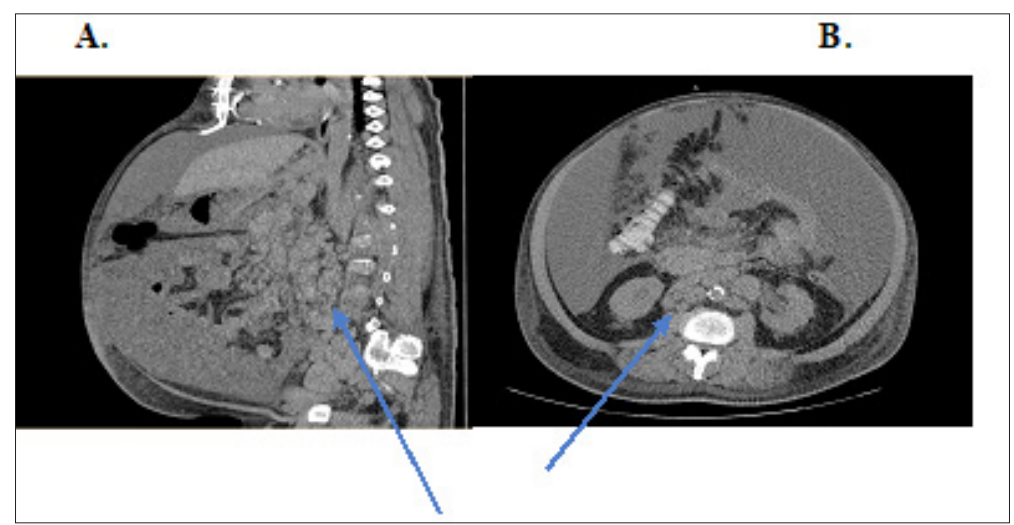

Figure 1: Chylous ascites in the peritoneum and retroperitoneal lymphadenopathy (arrows) on CT scan of the abdomen and pelvis (A) sagittal view; (B) axial view.

Table 1: Summary of laboratory results.

\begin{tabular}{|c|c|c|}
\hline Laboratory Measure & Value & Reference Range/Units \\
\hline Sodium & $131 \mathrm{mmol} / \mathrm{L}$ & $3.5-5.5 \mathrm{mmol} / \mathrm{L}$ \\
\hline Potassium & $6.9 \mathrm{mmol} / \mathrm{L}$ & $96-110 \mathrm{mmol} / \mathrm{L}$ \\
\hline Chloride & $101 \mathrm{mmol} / \mathrm{L}$ & $24-31 \mathrm{mmol} / \mathrm{L}$ \\
\hline Bicarbonate & $17 \mathrm{mmol} / \mathrm{L}$ & $5-13 \mathrm{units}$ \\
\hline Anion Gap & $13 \mathrm{units}$ & $5-25 \mathrm{mg} / \mathrm{dL}$ \\
\hline Blood Urea Nitrogen & $133 \mathrm{mg} / \mathrm{dL}$ & $0.61-1.24 \mathrm{mg} / \mathrm{dL}$ \\
\hline Creatinine & $5.26 \mathrm{mg} / \mathrm{dL}$ & $>60 \mathrm{~mL} / \mathrm{min}$ \\
\hline Glomerular Filtration Rate & $11 \mathrm{~mL} / \mathrm{min}$ & $2.5-4.6 \mathrm{mg} / \mathrm{dL}$ \\
\hline Phosphorus & $9.1 \mathrm{mg} / \mathrm{dL}$ & $4.0-8.0 \mathrm{mg} / \mathrm{dL}$ \\
\hline Uric Acid & $15.2 \mathrm{mg} / \mathrm{dL}$ & $3.5-5.5 \mathrm{~g} / \mathrm{dL}$ \\
\hline Albumin & $3.7 \mathrm{~g} / \mathrm{dL}$ & $0.2-1.3 \mathrm{mg} / \mathrm{dL}$ \\
\hline Bilirubin & $0.7 \mathrm{mg} / \mathrm{dL}$ & $91-200(\mathrm{iU}) / \mathrm{L}$ \\
\hline Lactate Dehydrogenase & $142(\mathrm{iU}) / \mathrm{L}$ & $13.2-17.5 \mathrm{gm} / \mathrm{dL}$ \\
\hline Hemoglobin & $11.4 \mathrm{gm} / \mathrm{dL}$ & $40.0-53.0 \%$ \\
\hline Hematocrit & $37.9 \%$ & $4.5-11.0 \mathrm{~K} / \mathrm{uL}$ \\
\hline White Blood Cell Count & $69.9 \mathrm{~K} / \mathrm{uL}$ & $140-450 \mathrm{~K} / \mathrm{uL}$ \\
\hline Platelets & $222 \mathrm{~K} / \mathrm{uL}$ & \\
\hline
\end{tabular}

Peritoneal fluid analysis yielded elevated peritoneal fluid WBC of $4,054 \mathrm{~K} / \mathrm{uL}$, triglycerides (TG) of $704 \mathrm{mg} / \mathrm{dL}$, and positive chylomicrons (Table 2). Three days later, he developed an increased build-up of ascites and underwent a second abdominal paracentesis with drainage of $3,500 \mathrm{~mL}$ cloudy amber fluid. Cytology showed mature lymphocytes. He subsequently went for a 
lymph node biopsy several days later; pathology showed numerous atypical lymphoid follicles, consistent with follicular lymphoma. Flow cytometry was positive for monotypic CD10+ monotypic B-cells. During this hospital course a bone marrow biopsy was performed which showed diffuse involvement (80\%) by an atypical lymphoid infiltrate of a hypercellular marrow ( $>90 \%$ ) with residual trilineage hematopoiesis, confirming follicular lymphoma (Figure 2). He started chemotherapy treatment with cyclophosphamide, doxorubicin, vincristine, prednisone and rituximab (R-CHOP) for stage IV follicular lymphoma during his admission and was later discharged to home with oncology follow-up outpatient. He underwent multiple therapeutic paracentesis after his initial paracentesis, and after his one session of dialysis on admission, his renal function improved without further hemodialysis sessions. He responded well to chemotherapy outpatient. Unfortunately, four months after this hospital admission, he suffered a witnessed cardiac arrest at home and Emergency Medical Services were called. When he arrived in the ED he was still undergoing cardiopulmonary resuscitation but ultimately return of spontaneous circulation was not achieved and he expired.

Table 2: Peritoneal fluid study results.

\begin{tabular}{|c|c|c|}
\hline Laboratory Measure & Value & Reference Range/Units \\
\hline Color & Pink & Clear to Pale Yellow \\
\hline Character & Milky & Clear \\
\hline Protein & $3.9 \mathrm{~g} / \mathrm{dL}$ & $0.3-4.0 \mathrm{~g} / \mathrm{dL}$ \\
\hline Albumin & $2.8 \mathrm{~g} / \mathrm{dL}$ & $3.5-5.5 \mathrm{~g} / \mathrm{dL}$ \\
\hline WBC Count & 4054, 86\% lymphocytes K/uL & $0-1000 \mathrm{~K} / \mathrm{uL}$ \\
\hline RBC Count & Too numerous to count & None \\
\hline Segmental Cell Count & $2 \%$ & $0-25 \%$ \\
\hline Triglycerides & $704 \mathrm{mg} / \mathrm{dL}$ & $<110 \mathrm{mg} / \mathrm{dL}$ \\
\hline Chylomicrons & Detected & Absent \\
\hline Fluid Culture & No Growth at 5 Days & No Growth at 5 Days \\
\hline
\end{tabular}

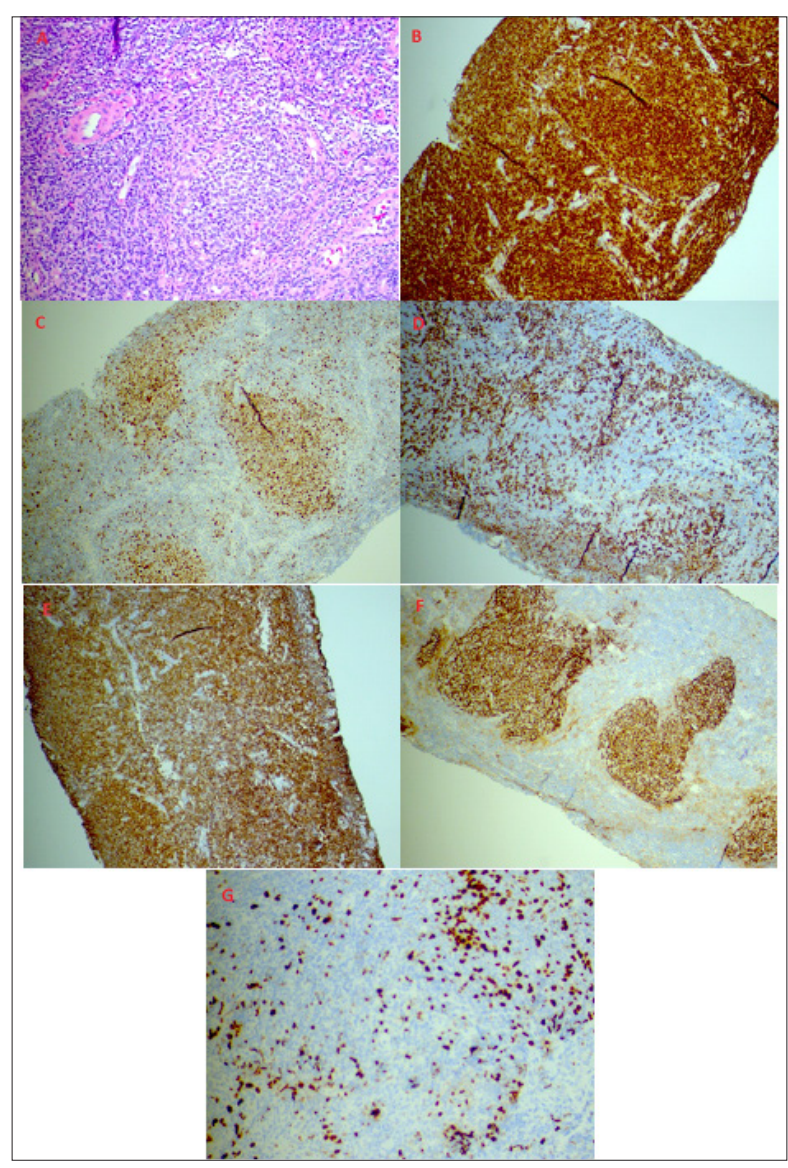

Figure 2: Lymph node biopsy demonstrating non-Hodgkin's lymphoma; Hematoxylin and eosin stain 20x view (A); BCL-2 immunostain (B); BCL-6 immunostain (C); CD3 immunostain (D); CD20 immunostain (E); CD21 immunostain (F); Ki-67 immunostain (G). 


\section{Discussion}

ACS is a rare but clinically significant manifestation in critical illness. ACS is defined by the World Society on Abdominal compartment Syndrome (WSACS) as intra-abdominal hypertension (IAH) with sustained IAP over $20 \mathrm{mmHg}$ [3]. ACS is usually preceded by IAH, defined as sustained end-expiratory abdominal pressure over $12 \mathrm{mmHg}$ (normal range 2 to $7 \mathrm{mmHg}$ ) in supine positioning with fully relaxed abdominal musculature [3]. While IAH is less likely associated with end-organ dysfunction, ACS is conversely one of the most severe outcomes of disease involving increased intraabdominal pressure. Although the precise incidence of ACS and IAH is poorly defined in the literature, few reports describe ACS occurring between $10 \%$ and $35 \%$ of non-injured critical patients, with a parallel incidence in post-operative or trauma patients [4-6]. In both subsets of patients, the presence of IAH or ACS has been associated with significantly increased mortality.

Physiologically, ACS and IAH involve increased pressure within a fixed volume of an anatomic compartment, in this case abdominal compartment, leading to decreased tissue perfusion due to impaired capillary, venous and arteriole blood flow [7]. Resulting cellular hypoxia causes lactic acidosis due to anaerobic processes. Increased upward pressure on the diaphragm prevents adequate ventilation, perfusion and impaired $\mathrm{CO}_{2}$ exchange, yielding a metabolic acidosis exacerbated by respiratory acidosis [7]. Grave manifestations of ACS include acute kidney injury due to decreased glomerular flow, decreased cardiac output due to compressed inferior vena cava, and poor perfusion of intestines due to decreased venous flow from splanchnic circulation [7]. If not addressed promptly, this series of events can lead to subsequent systemic acidosis and cardiovascular collapse.

There are many causes and risk factors leading to decreased abdominal wall compliance and increased volume of abdominal contents causing ACS, including burns, abdominal wall operations, large-volume crystalloid-based resuscitation ( $>5$ liters in 24 hours) and bowel edema, in addition to sepsis, trauma, advanced liver disease and portal hypertension [3,8-11]. Space-occupying lesions such as large tumors or ascites, hemoperitoneum, pancreatitis and severe ileus have also been involved in the development of IAH and ACS $[3,10,12]$. Few reports in the literature have described ACS as a complication of a progressive mass or serosanguinous ascites in lymphoma patients [13-15]. Upon performing a PubMed search including key words "abdominal compartment syndrome," nonHodgkin's lymphoma" and "chylous ascites" to date there is no report in the literature on ACS due to chylous ascites. Herein, we present the first.

Our unique case describes a 60-year-old male who presented with ACS secondary to large-volume chylous ascites in the setting of newly diagnosed stage IV follicular non-Hodgkin's lymphoma (NHL). In detail, our patient responded to large-volume paracentesis (13.5 liters) and chemotherapy with cyclophosphamide, doxorubicin, vincristine, prednisone and rituximab (R-CHOP). Early recognition of increased IAP and investigation of the etiology is essential in management before significant organ failure develops.
Serous effusions are common complications of malignant lymphomas. Presence of an effusion in lymphoma has been identified as a factor that adversely influences overall survival and portends a higher risk for relapse after chemotherapy. The most common effusion site in NHL and Hodgkin's lymphoma (HL) is pleural, with an incidence of $20-30 \%$, followed by more infrequent involvement of the peritoneal and pericardial cavities [16]. More exceedingly rare is the presence of chylous ascites.

Chylous ascites, a milky-appearing peritoneal fluid rich in triglycerides, develops from disruption of the lymphatic system during traumatic injury or from obstruction of lymphatic flow, in this case due to malignancy [17]. This obstruction causes leaking of chyle from dilated sub-serosal lymphatics into the peritoneal cavity, causing elevated pressures in the abdominal lymphatic system. This thus impairs intestinal absorptive capacity. In cases of acquired obstruction from trauma or surgery, the formation of a lymphoperitoneal fistula causes direct leakage of chyle [17]. Additionally, chylous ascites is associated with diminished water, electrolytes, immunoglobulins and lymphocytes which can predispose to organ dysfunction and increased susceptibility to infections [18].

There are several imaging modalities used to determine the extent of chylous fluid and to localize the leak or obstruction. Computed tomography (CT) of the abdomen is useful to identify pathologic intra-abdominal lymph nodes and masses and as well as to determine the extent of localization of fluid in cases of thoracic duct injury. Lymphangiography (LAG) is the gold standard imaging modality in cases of obstruction to localize the chyle leaks. In one analysis of 16 patients with chyle leaks who underwent LAG alone, the leak sites were identified in all cases [19]. However, LAG can be associated with complications including tissue necrosis, fat embolism or hypersensitivity due to contrast used [20]. Additional testing to define chylous ascites includes assessing TG levels in ascitic fluid, with a value above $200 \mathrm{mg} / \mathrm{dL}$ indicating chylous ascites [17,21-23]. Total protein content varies by underlying cause, ranging between 2.5 to $7.0 \mathrm{~g} / \mathrm{dL}$. The serum to ascites albumin gradient should also be calculated to determine if the ascites is related to portal hypertension or other causes, including malignancy $[24,25]$. Here, we emphasize the importance of prompt investigation of chylous ascites to provide timely and efficient treatment.

Appropriate treatment of ACS includes prompt normalization of the IAP to restore adequate visceral perfusion and to resolve the above-mentioned cardiopulmonary functional processes. There are several steps in the definitive management of ACS. Tense ascites, due to chyle in our patient case, can be treated by large-volume paracentesis. Management of severe forms of IAH, including highgrades III and IV defined as IAP $>21-25 \mathrm{mmHg}$ and IAP $>25 \mathrm{mmHg}$ respectively, usually requires emergent surgical decompression of the abdomen by decompressive laparotomy [7]. Conversely, causes of lower-grade IAH including burns, third-spacing of fluids into the abdominal cavity or a tight abdominal wall repair status post-surgical hernia repair can be treated with non-surgical means: paracentesis, neuromuscular blockade to reduce IAP as a 
temporizing measure, diuresis, or loosen the abdominal closure device [7].

In our case, both treating ACS via paracentesis and targeting NHL, the underlying cause of chylous ascites, with follicularlymphoma-directed therapy (R-CHOP) were crucial for clinical resolution. Treatment options for follicular lymphoma varies, as patients can live well without treatment for years. Stages I and II disease can be treated conservatively or with radiation therapy, while bulky stage II or stages III-IV disease can be treated with biologic agents of chemotherapy, with or without radiation therapy [26]. In refractory cases, surgical interventions including ligation of the thoracic duct or creation of a pleuroperitoneal shunt can be pursued [26]. Currently, there are no definitive guidelines for treatment of follicular lymphoma-related chylous effusions due to the rarity of this disease manifestation. Chylous ascites confers a poor prognosis, as seen in our patient with eventual cardiopulmonary collapse. Nevertheless, we aim to raise awareness on the importance of understanding the underlying pathophysiology that predisposes such patients to IAH to facilitate effective management strategies.

\section{Conclusion}

In this case report, we present a patient who was ultimately diagnosed with follicular lymphoma presenting as respiratory distress and acute renal failure secondary to stage IV follicular lymphoma. Our case illustrates a less commonly observed etiology of ACS in that it is 1) secondary to a malignancy and 2) due to chylous ascites rather than a mass. In our literature search, there are no cases to date that describe the development of ACS due to chylous ascites in the setting of malignancy. We thus present the first reported case. ACS, if unrecognized and untreated, can lead to rapid renal and cardiorespiratory compromise. Early diagnosis is key for treatment of this uncommon condition. The clinician should be aware that upon evaluating patients with abdominal distension and signs of hypoperfusion, such as end-organ damage, the diagnosis of ACS should be considered. In patients who do have ACS, treatment depends on the grade and can consist of abdominal decompression along with supportive measures. We thus suggest that chylous ascites should be included in the differential diagnosis and active work-up in patients with ACS, as this critical entity requires prompt recognition and treatment.

\section{Author's Contributions}

Marjan Alidoost: Case selection, planning and drafting the manuscript.

Conte GA: Contributed to discussion and edition of the manuscript.

Patel I: Contributed to case presentation part of the manuscript.

Farooq T: Contributed to the pathological slide review and discussion.

Dada 0: Contributed to collection and description of radiographic images.
Miskoff JA: Contributed in preparation and finalized the manuscript.

Levitt MJ: Contributed in manuscript revision and final approval.

\section{Acknowledgment}

The authors gratefully acknowledge the support of the Department of Pathology at Jersey Shore University Medical Center, Hackensack Meridian Health. We thank our patient who kindly gave his consent for this publication.

\section{References}

1. Crippa MW, Perumean JC (2019) Abdominal compartment syndrome. BMJ Best Practice.

2. Holodinsky JK, Roberts DJ, Ball CG, Blaser AR, Starkopf J, et al. (2013) Risk factors for intra-abdominal hypertension and abdominal compartment syndrome among adult intensive care unit patients: A systematic review and meta-analysis. Crit Care 17(5): R249.

3. Kirkpatrick AW, Roberts DJ, De Waele J, Jaeschke R, Malbrain ML, et al. (2013) Intra-abdominal hypertension and the abdominal compartment syndrome: Updated consensus definitions and clinical practice guidelines from the world society of the abdominal compartment syndrome. Intensive Care Med 39(7): 1190-1206.

4. Vidal MG, Ruiz Weisser J, Gonzalez F, Toro MA, Loudet C, et al. (2008) Incidence and clinical effects of intra-abdominal hypertension in critically ill patients. Crit Care Med 36(6): 1823-1831.

5. Balogh Z, McKinley BA, Cocanour CS, Kozar RA, Holcomb JB, et al. (2002) Secondary abdominal compartment syndrome is an elusive early complication of traumatic shock resuscitation. Am J Surg 184(6): 538543.

6. Balogh Z, McKinley BA, Holcomb JB, Miller CC, Cocanour CS, et al. (2003) Both primary and secondary abdominal compartment syndrome can be predicted early and are harbingers of multiple organ failure. J Trauma 54(5): 848-859.

7. Maluso P, Olson J, Sarani B (2016) Abdominal compartment hypertension and abdominal compartment syndrome. Crit Care Clin 32(2): 213-222.

8. Wilson A, Longhi J, Goldman CD, Mcnatt S (2010) Intra-abdominal pressure and the morbidly obese patients: The effect of body mass index. J Trauma 69(1): 78-83.

9. Hering R, Wrigge H, Vorwerk R, Brensing K, Schroder S, et al. (2001) The effects of prone positioning on intraabdominal pressure and cardiovascular and renal function in patients with acute lung injury. Anesth Analg 92(5): 1126-1231.

10. Blaser AR, Parm P, Kitus R, Starkopf J (2011) Intra-abdominal hypertension and gastrointestinal symptoms in mechanically ventilated patients. Crit Care Res Pract 982507.

11. Kasotakis G, Duggan M, Li Y, O’Dowd D, Baldwin K (2013) Optimal pressure of abdominal gas insufflation for bleeding control in a severe swine splenic injury model. J Surg Res 184(2): 931-936.

12. Blaser AR, Parm P, Kitus R, Starkopf J (2011) Risk factors for intraabdominal hypertension in mechanically ventilated patients. Acta Anaesthesiol Scand 55(5): 607-614.

13. Bozkurt MA, Temizgonul KB, Kones O, Alis H (2012) A rare reason of abdominal compartment syndrome: Non-Hodgkin lymphoma. J Korean Surg Soc 83(4): 242-245.

14. Egyed E, Heiss MM, Wappler F, Sakka S (2019) Successful treatment of abdominal compartment syndrome with chemotherapy in a patient with a newly diagnosed Burkitt lymphoma. J Crit Care 51: 26-28. 
15. Hendrick JM, Kaste SC, Tamburro RF, Hoffer FA, Onciu M, et al. (2006) Abdominal compartment syndrome in a newly diagnosed patient with Burkitt lymphoma. Pediatr Radiol 36(3): 254-257.

16. Das DK (2006) Serous effusions in malignant lymphomas: A review. Diagn Cytopathol 34(5): 335-347.

17. Cardenas A, Chopra S (2002) Chylous ascites. Am J Gastroenterol 97(8): 1896-1900.

18. Bhardwaj R, Vaziri H, Gautam A, Ballesteros E, Karimeddini D, et al. (2018) Chylous ascites: A review of pathogenesis, diagnosis and treatment. Journal of Clinical and Translational Hepatology 6(1): 105113.

19. Deso S, Ludwig B, Kabutey NK, Kim D, Guermazi A (2012) Lymphangiography in the diagnosis and localization of various chyle leaks. Cardiovasc Intervent Radiol 35(1): 117-126.

20. Pui MH, Yueh TC (1998) Lymphoscintigraphy in chyluria chyloperitoneum and chylothorax. J Nucl Med 39(7): 1292-1296.
21. Press OW, Press NO, Kaufman SD (1982) Evaluation and management of chylous ascites. Ann Intern Med 96(3): 358-364.

22. Runyon BA, Hoefs JC, Morgan TR (1988) Ascitic fluid analysis in malignancy-related ascites. Hepatology 8(5): 1104.

23. Jungst D, Gerbes AL, Martin R, Paumgartner G (1986) Value of ascitic lipids in the differentiation between cirrhotic and malignant ascites. Hepatology 6(2): 239-243.

24. Runyon BA, Montano AA, Akriviadis EA, Antillon MR, Irving MA, et al. (1992) The serum-ascites albumin gradient is superior to the exudatetransudate concept in the differential diagnosis of ascites. Ann Intern Med 117(3): 215-220.

25. Runyon BA (1994) Care of patients with ascites. N Engl J Med 330(5): 337-342.

26. Luminari S, Bellei M, Biasoli I, Federico M (2012) Follicular lymphomatreatment and prognostic factors. Rev Bras Hematol Hemoter 34(1): 5459. 